\title{
Helminth infections: the great neglected tropical diseases
}

\author{
Peter J. Hotez, ${ }^{1}$ Paul J. Brindley, ${ }^{1}$ Jeffrey M. Bethony, ${ }^{1}$ Charles H. King, ${ }^{2}$ \\ Edward J. Pearce, ${ }^{3}$ and Julie Jacobson ${ }^{4}$
}

\begin{abstract}
1Department of Microbiology, Immunology, and Tropical Medicine, George Washington University, Washington, DC, USA.
${ }^{2}$ Center for Global Health and Diseases, Case Western Reserve University School of Medicine, Cleveland, Ohio, USA. ${ }^{3}$ Department of Pathobiology,

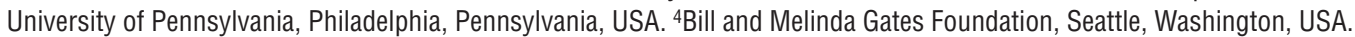

\begin{abstract}
Helminths are parasitic worms. They are the most common infectious agents of humans in developing countries and produce a global burden of disease that exceeds better-known conditions, including malaria and tuberculosis. As we discuss here, new insights into fundamental helminth biology are accumulating through newly completed genome projects and the nascent application of transgenesis and RNA interference technologies. At the same time, our understanding of the dynamics of the transmission of helminths and the mechanisms of the Th2-type immune responses that are induced by infection with these parasitic worms has increased markedly. Ultimately, these advances in molecular and medical helminth biology should one day translate into a new and robust pipeline of drugs, diagnostics, and vaccines for targeting parasitic worms that infect humans.
\end{abstract}

\section{Introduction}

Helminths (the word is derived from the Greek meaning "worms"; ref. 1) have plagued humans since before the era of our earliest recorded history. The eggs of intestinal helminths can be found in the mummified feces of humans dating back thousands of years $(2-4)$, and we can recognize many of the characteristic clinical features of helminth infections from the ancient writings of Hippocrates, Egyptian medical papyri, and the Bible (2-4). These same helminthiases markedly altered the course of modern twentieth century world history $(2,4-7)$, especially in China during the Cold War, when the schistosome was known as "the blood-fluke that saved Formosa” (7) because acute schistosomiasis sickened Mao's troops and aborted their amphibious assault of Taiwan (historically known as Formosa) just long enough for American ships to enter the Straits of Taiwan (5-7).

There are two major phyla of helminths. The nematodes (also known as roundworms) include the major intestinal worms (also known as soil-transmitted helminths) and the filarial worms that cause lymphatic filariasis (LF) and onchocerciasis, whereas the platyhelminths (also known as flatworms) include the flukes (also known as trematodes), such as the schistosomes, and the tapeworms (also known as the cestodes), such as the pork tapeworm that causes cysticercosis (Table 1). In 1947, Norman Stoll published a landmark paper entitled "This wormy world," in which he set out to estimate the number of people infected with helminths worldwide (8). Over the last 60 years, several estimates have confirmed Stoll's initial observation that hundreds of millions of people harbor parasitic worms (9-11). Today, it is estimated that approximately one-third of the almost three billion people that live on less than two US dollars per day in developing regions of sub-Saharan Africa, Asia, and the Americas are infected with one or more helminth (12, 13). The most common helminthiases are those caused by infec-

Nonstandard abbreviations used: LF, lymphatic filariasis; MDA, mass drug administration.

Conflict of interest: P.J. Hotez is an inventor on two international patent applications for a hookworm vaccine. P.J. Hotez and J.M. Bethony received funding from the Bill and Melinda Gates Foundation through the Sabin Vaccine Institute. The remaining authors have declared that no conflict of interest exists.

Citation for this article: J. Clin. Invest. 118:1311-1321 (2008). doi:10.1172/JCI34261. tion with intestinal helminths, ascariasis, trichuriasis, and hookworm, followed by schistosomiasis and LF (Table 1). Practically speaking, this means that the inhabitants of thousands of rural, impoverished villages throughout the tropics and subtropics are often chronically infected with several different species of parasitic worm; that is, they are polyparasitized $(12,13)$.

For reasons not well understood, compared with any other age group, school-aged children (including adolescents) and preschool children tend to harbor the greatest numbers of intestinal worms and schistosomes and as a result experience growth stunting and diminished physical fitness as well as impaired memory and cognition (14). These adverse health consequences combine to impair childhood educational performance, reduce school attendance (15), and account for the observation that hookworm (and presumably other diseases caused by parasitic worms) reduces future wage-earning capacity (16). Hookworm and schistosomiasis are also important diseases during pregnancy, causing neonatal prematurity, reduced neonatal birth weight, and increased maternal morbidity and mortality (17). Among some adult populations living in impoverished areas of developing countries, onchocerciasis is a leading cause of blindness and skin disease, while LF is a major cause of limb and genital deformities. LF and onchocerciasis, together with hookworm and schistosomiasis, are also major determinants of reduced worker productivity (4, $18,19)$. Such chronic, disabling, and often disfiguring effects of helminths translate into enormous poverty-promoting effects and represent a major reason why poor people remain mired in a downward cycle of destitution (19).

Adding to the global morbidity that results from human helminth infections are the observations that they have both direct and indirect effects on malaria and HIV/AIDS in developing countries. In sub-Saharan Africa and elsewhere, helminthiases are frequently coendemic with malaria $(12,20-22)$ and HIV/AIDS (12, 23-26). Indeed, it is not uncommon for an individual to be coinfected with the malaria-causing parasite and one or more parasitic worm (22), or HIV and one or more parasitic worm $(25,26)$. Such coinfections have additive effects, such as severe anemia (21), and synergistic effects, such as increased transmission of the malariacausing parasite, HIV, and/or increased susceptibility to infection 


\section{Table 1}

The major human helminthiases and their global prevalence and distribution

\begin{tabular}{|c|c|c|c|}
\hline Disease & Major etiologic agent & Global prevalence & Regions of highest prevalence \\
\hline \multicolumn{4}{|l|}{ Soil-transmitted nematodes } \\
\hline Ascariasis & Ascariasis lumbricoides (roundworm) & 807 million & Developing regions of Asia, Africa, and Latin America \\
\hline Trichuriasis & Trichuris trichiura (whipworm) & 604 million & Developing regions of Asia, Africa, and Latin America \\
\hline Hookworm & Necator americanus; Ancylostoma duodenale & 576 million & $\begin{array}{l}\text { Developing regions of Asia, Africa, and Latin America } \\
\text { (especially areas of rural poverty) }\end{array}$ \\
\hline Strongyloidiasis & Strongyloides stercoralis (thread worm) & 30-100 million & $\begin{array}{l}\text { Developing regions of Asia, Africa, and Latin America } \\
\text { (especially areas of rural poverty) }\end{array}$ \\
\hline \multicolumn{4}{|l|}{ Filarial nematodes } \\
\hline LF & Wuchereria bancrofti; Brugia malayi & 120 million & $\begin{array}{c}\text { Developing regions of India, Southeast Asia, } \\
\text { and sub-Saharan Africa }\end{array}$ \\
\hline Onchocerciasis (river blindness) & Onchocerca volvulus & 37 million & Sub-Saharan Africa \\
\hline Loiasis & Loa loa & 13 million & Sub-Saharan Africa \\
\hline Dracunculiasis (guinea worm) & Dracunculus medinensis & 0.01 million & Sub-Saharan Africa \\
\hline \multicolumn{4}{|l|}{ Platyhelminth flukes } \\
\hline \multicolumn{2}{|r|}{$\begin{array}{l}\text { Schistosoma haematobium; } \\
\text { Schistosoma mansoni; } \\
\text { Schistosoma japonicum (blood flukes) }\end{array}$} & 207 million & $\begin{array}{c}\text { Sub-Saharan Africa } \\
\text { Sub-Saharan Africa and Eastern Brazil } \\
\text { China and Southeast Asia }\end{array}$ \\
\hline Food-borne trematodiases & $\begin{array}{l}\text { Clonorchis sinensis (liver fluke); } \\
\text { Opisthorchis viverinni (liver fluke); } \\
\text { Paragonimus spp. (lung flukes); } \\
\text { Fasciolopsis buski (intestinal fluke); } \\
\text { Fasciola hepatica (intestinal fluke) }\end{array}$ & $>40$ million & Developing regions of East Asia \\
\hline \multicolumn{4}{|l|}{ Platyhelminth tapeworms } \\
\hline Cysticercosis & Taenia solium (pork tapeworm) & $\begin{array}{l}0.4 \text { million } \\
\text { (Latin America only) }\end{array}$ & $\begin{array}{c}\text { Developing regions of Asia, Latin America, } \\
\text { and sub-Saharan Africa }\end{array}$ \\
\hline
\end{tabular}

with these pathogens as well as cause an exacerbated progression of these two killer diseases (12, 20-26).

The high medical, educational, and economic burden of helminth infections, together with their coendemicity with malaria and AIDS, provides an important rationale for launching a global assault on parasitic worms (13). However, the tools we currently have for controlling worm infections are limited; of the 1,556 new chemical entities marketed between 1975 and 2004, only four drugs - albendazole, oxamniquine, praziquantel, and ivermectin - were developed to treat helminthiases $(4,27)$. Together with diethylcarbamazine (developed in the first half of the twentieth century) and mebendazole, these drugs represent almost our entire pharmacopeia for combating the most common infections in the world. The dearth of available anthelminthic agents partly reflects the very modest commercial markets for drugs targeting human helminth infections and partly reflects how remarkably little we know about the unique biochemical metabolism of parasitic worms and the mechanisms by which worms evade human host defenses, establish chronic infections, and cause adverse maternal and child health (14). Indeed, the diseases caused by infection with helminths are considered neglected tropical diseases, and the study of these diseases receives less than $1 \%$ of global research dollars (4). Despite this, as we discuss here, recent advances by molecular and immunological helminthologists have indicated that helminths are a rich source of interesting molecules that could lead to innovation for almost all aspects of biomedicine. We hope that such information might one day translate into the development of new drugs, diagnostics, and vaccines to combat infection with helminths as well as influence the development of new therapeutics for other human illnesses.

\section{Epidemiology of helminth infections}

With the exception of Strongyloides stercoralis, helminths do not replicate within the human host. This fundamental aspect of helminth biology establishes a set of transmission dynamics quite different than those for viruses, bacteria, fungi, and protozoa. For example, prevalence, which is the proportion of persons in a defined population at a given time point infected with the helminth (28), is seldom used as the only measure to assess the epidemiological situation for that helminth infection, because morbidity is associated with the number of worms infecting the host (i.e., the worm burden) rather than the absence or presence of infection. Prevalence is commonly combined with worm burden (also referred to as the "intensity of infection"), which is commonly measured by the number of eggs per gram (EPGs) of feces for intestinal helminths and schistosomes (29). Based on EPGs and their association with morbidity, individuals are classified into categories of light, moderate, and heavy infection by the WHO (30). Furthermore, in the case of soil-transmitted helminths, the WHO recommends use of both prevalence and intensity of infection to classify communities into transmission categories - category I (high), category II (medium), and category III (low). These transmission categories are assigned according to both the number of heavily infected people in the community (greater or less than $10 \%$ ) and the prevalence of infection (greater or less than $50 \%$ ). For example, a community with greater than $50 \%$ prevalence but 
less than $10 \%$ heavy infection would be considered a category II transmission community. The WHO uses this information in algorithms to determine the type of mass treatment a community should receive. For filarial helminths, the transmission status in a community is determined by assessing the number of people with blood-circulating microfilariae or microfilarial antigen; in the case of onchocerciasis, it is determined by counting the number of microfilariae in a skin biopsy or even counting the number of palpable nodules (30). It important to note that the quantitative assessment of worms provides no information on whether the infection represents either a recent or long-term infection nor does it indicate either the length or extent of exposure to infection (i.e., an individual residing near a source of transmission for many years may not necessarily have high egg count despite extensive exposure). There are several key determinants underlying the epidemiology of helminth infections.

Environment. Climate and topography are crucial determinants of the distribution of helminth infections (31). Helminths transmitted by vectors are limited to landscapes in which host and vector come together in the same habitat, resulting highly focal distribution. For example, the distribution of schistosomiasis reflects the biotic and abiotic features (i.e., climatic, physical, and chemical factors) that affect the survival and development of the snail vector (32). In the case of onchocerciasis, the distribution and incidence of the disease are limited by biogeographic variations favorable to exposure to the blackfly vectors $(33,34)$. Soil-transmitted helminths are highly affected by surface temperature (35), altitude, soil type, and rainfall $(36,37)$.

Heterogeneity. Heterogeneity in the worm burden among different individuals infected with the same helminth is a hallmark feature of helminth epidemiology (38). A consequence of such heterogeneity is the aggregated distribution of helminth infection in endemic communities, such that a small proportion of hosts are rapidly, frequently, and/or heavily infected $(38,39)$. For example, $70 \%$ of the worm burden occurs in $15 \%$ of the infected individuals at a given time point (40). The aggregated distribution of helminth infection has led some to hypothesize that certain "wormy" people are "predisposed" to heavy infection from as yet undefined genetic, immunogenetic, ecological, behavioral, and social factors (41). Predisposition refers to studies in which intensity of infection prior to anthelminthic treatment positively correlates with intensity of reinfection 12-24 months after treatment (41). The bases of both heterogeneity and predisposition to helminth infection have yet to be fully elucidated. However, among the major factors under consideration are age, household clustering, and genetics.

Age dependency. Much epidemiologic research has focused on heterogeneity in the intensity of helminth infection by age (42). Changes with age in the average intensity of infection tend to be convex, rising in childhood and declining in adulthood. For Ascaris lumbricoides and Trichuris trichiura, the heaviest and most frequent infections are in children aged $5-15$ years, with a decline in intensity and frequency in adulthood (43). Similarly, for all the major schistosomes, the heaviest and most frequent infections are in older children aged 10-15 years (44). In contrast, hookworm frequently exhibits a steady rise in intensity of infection with age, peaking in adulthood (45). Similarly, the pathologic events that occur with filarial infections also predominate in adulthood.

Some of the strongest evidence for protective immunity to human helminth infection has come from epidemiological observations of a "peak shift" in prevalence and intensity of infection with age
(46). Briefly, if age-infection data are compared across host populations, the peak level of infection intensity (e.g., EPGs for intestinal helminths) is higher and occurs in younger individuals when transmission is also higher, but the peak intensity of infection is lower and occurs in older individuals when transmission is lower (46). This shift in the peak level of infection intensity and the age at which this peak occurs is consistent with mathematical models that assume aradually acquired protective immunity, an interpretation supported by experimental studies in animals (46).

Household clustering. Evidence for household clustering of infected individuals exists for most diseases caused by infection with a helminth, including ascariasis (47), trichuriasis (47), and strongyloidiasis $(48,49)$. This clustering can persist through time, as shown by familial predisposition to heavy infection with Ascaris lumbricoides and Trichuris trichiura in Mexico (50). Household aggregation of lymphatic filarial infection (individuals with LF and/or microfilaraemia) has been described in India and Polynesia $(51,52)$. In one study of schistosomiasis, shared household accounted for $22 \%$ of the variance in S. mansoni egg counts (53). There is also some evidence for a similarity in antibody isotype level among family members for crude schistosome antigen extracts, reflecting the degree to which this phenotype might be influenced by genetic factors (54).

Genetics. For a number of species of parasitic worms, it has been established that the intensity of infection is a heritable phenotype (55). The most advanced research program investigating the identity of host genes that influence helminth infection involves schistosomiasis. The first genome scan for a helminth infection identified linkage of intensity of infection with S. mansoni in a Brazilian population to the chromosomal region 5q31-q33 (56), and subsequent confirmation of this link was established in a Senegalese population (57). The 5q31-q33 region includes loci for numerous immune response genes, including those encoding the Th2 cytokines IL-3, IL-4, IL-5, IL-9, and IL-13, interferon regulatory factor 1, colony-stimulating factor 2, colony-stimulating factor 1 receptor, and the IL-12/IL-23 p40 subunit (55). Genes controlling IgE production, asthma, malaria parasitaemia, and inflammatory bowel disease, among others, have also been mapped to this region of chromosome 5 , although the causative polymorphisms have not yet been identified.

Polyparasitism. Finally, an increasing number of studies of helminth epidemiology have shown that it is common for individuals to be infected with more than one species of helminth $(21,58-63)$. There is also evidence suggesting synergism and antagonism in concurrent intestinal nematode and schistosome infections (62-64) as well as filarial nematode infection and soil-transmitted helminth infections (65). A number of epidemiological studies have indicated that individuals infected with multiple species of helminth often harbor heavier infections than individuals infected with a single helminth species (58-61). An important consequence of simultaneous infection with the parasites that cause hookworm, schistosomiasis, and malaria is severe anemia $(21,66)$. It has also been speculated that helminth infections may adversely influence host immune responses to the malaria-causing parasite and other pathogens (20).

\section{Burden of helminthiases}

Most helminth infections, if left untreated, result in multi-year, chronic inflammatory disorders that cause both concurrent and delayed-onset pathology to the afflicted human host (67-69). In addition to the overt and dramatic effects of blindness and elephantiasis in individuals with onchocerciasis and LF, respec- 
tively, it is now appreciated that chronic helminth infections are also linked to more insidious persistent health conditions such as anemia, growth stunting, protein-calorie undernutrition, fatigue, and poor cognitive development (68). These seemingly subtle and often overlooked morbidities are very important because of the high prevalence of helminthiases in the rural developing world, in which any health impairment is substantially magnified in terms of degradation of individual patient performance status (70).

Initially, in childhood, it is the presence of helminth infection and the intensity of infection that determine the risk for disease formation. It is also true that for many of the tissue-invasive helminths, such as the schistosomes and filariae, tissue damage can continue into later adult life, with disease persisting and even increasing long after the infection is cleared. As such, measures of infection prevalence do not capture the prevalence of infectionassociated disease, particularly in adult life. Conditions such as elephantiasis, which occurs in individuals with LF; visual impairment, which occurs in individuals with onchocerciasis; periportal fibrosis and hypertension, which occur in individuals with intestinal schistosomiasis; biliary obstruction, cholangitis, and cholangiocarcinoma, which occur in individuals with food-borne trematodiasis; and urinary obstruction and bladder cancer, which occur in individuals with urinary schistosomiasis, are potentially the most life-threatening consequences of helminth infections. Although most likely to contribute to hospitalization and to cause mortality, these advanced outcomes are rare when compared to the disease burden of the average patient, which is characterized by the subacute morbidities detailed earlier.

The temporal lag between initial high-intensity childhood infection and the delayed onset of "classical" parasite-associated pathologic findings have led to a serious underappreciation of the day-to-day burden of helminthic diseases. In international health assessments based on the disability-adjusted life year (DALY) metric (71), DALY calculations are weighted to stress diseases prevalent among adults in the $20-40$ age group, and therefore diseases arising primarily in childhood carry far less weight (72). In the current Global Burden of Disease (GBD) assessments by the WHO, it is not clear whether prevalence of infection per se was used to gauge the disease burden of helminths or the more appropriate duration of infection-associated pathology, which is often irreversible. The DALY disability weights assigned to specific helminth infections were developed by nonpatient committees based on disease scenarios that did not reflect what we now appreciate as the full spectrum of helminth infection-associated pathology (71). Although efforts are underway to revise the GBD assessments (73), it is important that we do not underestimate the substantial disease burden of more than one billion individuals worldwide who are affected by helminth infections.

A new focus for assessing the health burden related to helminthic diseases has been the adoption of patient-based quality-of-life (QoL) interview techniques for assessing disease burden across many international settings $(74,75)$. Although health-related QoL is in some sense a cultural construct, and subjective adaptation to physical impairments is likely to vary from region to region, certain universal features of disease impact can be identified. Implementation of QoL assessment for most prevalent diseases, and the subsequent use of quality-adjusted life years (QALYs) for comparison of disease impacts (76), is likely to provide the most useful comparison of disease burdens across different societies and their economies.

\section{Helminth immunobiology}

The reduction in the intensity of some human helminth infections with age might be indicative of host immunity. Immune responses to helminths are intriguing not only from the perspectives of understanding protective immunity and immunopathology, but also because a major branch of the mammalian immune system, type 2 immunity, seems to have evolved specifically to deal with this class of pathogens. Type 2 immunity involves the rapid activation and engagement of cells of both the innate (eosinophils and basophils) and adaptive (CD4 ${ }^{+} \mathrm{T}$ cells that commit to the Th2 pathway) immune systems (77). Cells of both the innate and adaptive immune systems that are involved in type 2 immunity share the ability to synthesize the core type 2 cytokine IL-4, which mediates (both directly and indirectly) the reactions that historically have been considered to be symptomatic of helminth infection such as IgE production, eosinophilia, and changes in the physiology of target organs (e.g., the intestine and lungs) that are associated with goblet cell hyperplasia and smooth muscle contraction (78).

Based on findings from studies of infections in humans and mouse models of helminth infections, we know that, depending on the infection in question, type 2 immune responses can prevent the survival of infecting parasites during a homologous secondary infection (79), expel adult parasites from the gut (78), allow host survival in a setting where the immune response cannot clear the parasites (80), and/or mediate pathological fibrotic responses (81). Fibrosis has its origins in the wound-healing responses that must be required on an ongoing basis in animals chronically infected with pathogens that cause large amounts of tissue damage, such as most helminths (82).

In key ways, our understanding of type 2 immunity has lagged behind that of type 1 immunity (which encompasses Th1 cells) and even of the more recently defined Th17 cells and Tregs. In each of these cases, a set of cytokines made by cells of the innate immune system, often in response to the ligation of TLRs by a component of a pathogen, strongly promotes the development of defined $\mathrm{CD}^{+} \mathrm{T}$ cell lineages that in productive immune responses are appropriate to the particular situation. Thus, we know that IFN- $\gamma$ and IL-12 can promote Th1 responses; TGF- $\beta$, IL- 6 , and IL-23 can promote Th17 cell development; and TGF- $\beta$ alone plays a role in driving Treg development $(83,84)$. Although we know that IL-4 and IL-13 are important for Th2 cell development, the production of these cytokines by innate immune cells seems to play little part in promoting the initiation of Th 2 responses, and it has been clear that other crucial, yet ill-defined, conditions are important in this process. Recent work in type 2 immunity, largely involving excellent mouse models of human helminthiases and studies of allergy and asthma, which share with helminth infections an association with type 2 immune responses, has begun to shed light on some of these issues. For example, it is now clear that in addition to IL-4, IL-13, IL-5, IL-9, and IL-10, Th2 cells can make IL-25 and IL-31, and that these two cytokines can have crucial roles in promoting and/or regulating Th 2 immune responses $(85,86)$. There have also been substantial developments in understanding how the induction of type 2 immune responses is influenced by other cells, specifically through the recognition that thymic stromal lymphopoietin (TSLP), synthesized by epithelial cells, can condition dendritic cells to promote Th2 cell development (87). Lastly, there has been considerable progress in identifying genes that are expressed in response to stimulation by cytokines produced by Th2 cells and in defining the roles of the products of these genes as effectors of 
Th2 immunity as well as inducers and regulators of Th2 immune responses. Among these gene products are intelectins, arginases, resistin-like molecules (RELMs), and chitinases (88). Indeed, the coordinated production of members of the latter three classes of molecules by macrophages exposed to IL-4 and/or IL-13 is a feature of the "alternative activation" pathway executed by these cells during helminth infections. This is an area of great current interest (89), especially since recent reports have highlighted the protective effects of these cells during helminth infections $(80,90)$.

How more recently discovered components of type 2 immune responses, such as IL-25 and IL-31, participate in host protective and immunopathologic processes is only just beginning to be examined using helminth infections in the mouse (91-94), and little has been done as of yet to explore these pathways in humans infected with helminths. This is a target area for emphasis in immunologic research on helminths in the near future, both in terms of increasing our understanding of the basic immunobiology of infections with these parasites, but also and more importantly, as a means to improve attempts directed toward the rational development of vaccines and immunotherapeutics.

Many helminthiases are chronic diseases, and it remains unclear how the Th2 immune response is regulated during the course of these long-term infections, during which antigen loads remain high and pathology is, at least in part, immune mediated (95). There is great interest in the possibility that Tregs play a role in these processes, perhaps in combination with unusual immunoregulatory helminth-derived products (96), and that such regulation underlies the reverse correlations between infection with a helminth and asthma, allergy, and certain autoimmune diseases that have been reported in a growing number of studies (something that is known as the hygiene hypothesis) $(97,98)$. This remains a key area for further exploration, not only in the hope that understanding the regulatory response will allow intervention to enhance immune responses and decrease infection, but also because of the potential therapeutic use of helminth regulatory strategies for other disorders. Indeed, understanding how helminth infections regulate inflammation could promote new approaches for the development of therapeutics for a wide variety of inflammatory conditions.

\section{Helminth genomics, postgenomics, and gene manipulation}

Deciphering the genetic code of helminth parasites will allow us to understand the fundamental nature of these pathogens and the pathogenesis of the diseases that they cause. Genetic manipulation of helminth genomes, including transgenesis approaches, offers a means to determine the importance of specific helminth genes in disease pathogenesis, including those that could be targeted by novel therapeutic interventions.

Genomics. Helminth parasites have large complex genomes. In general, for both nematodes and platyhelminths, genome size ranges from approximately 50 to $500 \mathrm{Mb}$, with up to 20,000 proteinencoding genes. This genome size is comparable to that of insects. Several helminth parasites have become the focus of endeavors aiming to determine entire genome sequences. Information from these sequencing projects will facilitate determination of function and importance of genes or groups of genes in cellular and biochemical pathways and their role in the host-parasite relationship.

It is anticipated the complete genomes of Schistosoma mansoni and Schistosoma japonicum will be reported within the next year (99), fol- lowing the release recently of reasonably comprehensive descriptions of their transcriptomes $(100,101)$ and the proteome of S. japonicum (102). Schistosomes have sizeable genomes. The haploid genome of S. mansoi is estimated to be approximately $300 \mathrm{Mb}$, arrayed on seven pairs of autosomes and one pair of sex chromosomes (103). For comparison, this genome size is about ten times that of the genome of the malaria-causing parasite Plasmodium falciparum and one-tenth the size of the human genome. Schistosoma haematobium and S. japonicum, the other major schistosome species that parasitize humans probably have a genome size similar to that of S. mansoni, based on the similarity of their karyotypes (103). The S. mansoni genome is AT-rich (60\%-70\% AT in the euchromatin), replete with repetitive sequences. The approximately 13,000 protein-encoding genes include several, sometimes numerous, introns ranging in size from small to very large. The complexity of the proteome is expanded by the presence of single nucleotide polymorphisms, trans-splicing of a subset of the transcriptome, and alternative splicing of some genes (reviewed in ref. 104).

The draft genome sequence of the filarial nematode parasite Brugia malayi was reported recently (105). This was a landmark in human parasitology as it represented the first genome sequence of a parasitic helminth to be deciphered. B. malayi has a genome of $90 \mathrm{Mb}$, with a compliment of 14,500-17,800 protein-encoding genes. As in the free-living nematode Caenorhabditis elegans and many bacteria, a number of the B. malayi genes are organized into operons - units of transcription wherein several genes are regulated by the same promoter, thereby enabling the cell or organism to facilitate global gene expression in response to stimuli and environmental conditions.

The genome sequence of no parasitic nematode other than B. malayi has been completed. However, the draft genome sequence for B. malayi offers a broad foundation for rational intervention for both filariae and parasitic nematodes at large. Comprehensive analyses of the transcriptomes and genomes of a number of other medically important parasites, including hookworms and Trichinella spiralis, are in progress $(106,107)$. Through both genomics and more conventional biochemical methodologies, bioactive proteins with remarkable therapeutic potential have been identified and isolated from hookworms and other nematodes (reviewed in ref. 108). These include a novel factor VIIa/tissue factor inhibitor and a neutrophil inhibitory factor, which have undergone clinical trials with beneficial and promising outcomes for human thrombotic disease (109-112). These successes serve to emphasize that genomic studies of the parasitic helminths are likely to lead to new treatments for disease, including diseases not directly caused by helminth pathogens.

Gene manipulation by RNA interference. Until recently, gene manipulation approaches had not been seriously considered as tools to learn more about helminth-encoded proteins that might be intervention targets. This situation is now changing in response to the availability of genome sequences and other advances. RNAi technology has revolutionized investigation of the role and importance of genes in model organisms such as C. elegans. Methods developed for RNAi in C. elegans are now being deployed in schistosomes and some parasitic nematodes. Skelly and coworkers established the feasibility of experimental RNAi in S. mansoni by describing knockdown of the gut-associated cysteine protease cathepsin B by soaking larval schistosomes in dsRNA that effects RNAi (113). Krautz-Peterson et al. extended these studies by comparing and optimizing RNAi methodology (114). They reported that squarewave electroporation was dramatically more efficient than either 
soaking the parasites in an equivalent dsRNA dose or soaking them in an equivalent dsRNA dose in the presence of liposomes. They also demonstrated that small, interfering RNAs were as effective as longer dsRNAs. Other reports have confirmed the utility of RNAi in schistosomes to address gene function and importance (115-117). Indeed, RNAi has been used to demonstrate a crucial role for S. mansoni inhibin/activin (SmInAct; a member of the TGF- $\beta$ receptor family) in embryogenesis; knockdown of SmInAct expression in eggs aborts their development (118). Given that eggs are responsible for the pathology of schistosomiasis, this protein is a potential therapeutic intervention target. As gene silencing by RNAi in schistosomes has been reported to deliver a specific effect, with no obvious or widespread nonspecific effects on nontarget genes, gene manipulation by RNAi might have wide applicability and promote functional schistosome genomics. The latter are expected to improve our knowledge of the function of schistosome gene products and lead to new interventions for treatment and control.

In contrast to work with schistosomes, RNAi in parasitic nematodes has had limited success or has been unsuccessful $(119,120)$. Given the importance of RNAi in revolutionizing our understanding of the biology of the C. elegans, this overall lack of success for parasitic nematodes has so far been a disappointment for the molecular helminthology community. The physiological basis for the poor performance of RNAi in parasitic nematodes might relate to an absence of pivotal RNAi pathway components, as evidenced by the absence in $B$. malayi of the gene encoding the Sid-1 membrane channel that is involved in dsRNA uptake $(105,121)$.

Transgenesis. Gain-of-function approaches with reporter transgenes, for example transgenes encoding either jellyfish GFP or firefly luciferase, have also been investigated in parasitic worms. Monitoring the expression of the reporter protein provides information on the progress of the transgenesis procedures. In parasitic nematodes, reporter gene activity driven by exogenous and endogenous gene promoters has been demonstrated in B. malayi, S. stercoralis, and Parastrongyloides trichosuri (122-124). In addition, heritable transmission of plasmid-based transgenes has been reported $(123,124)$. Similar findings have been described in schistosomes, and stage- and tissue-specific expression of schistosome genes has been investigated using reporter transgenes driven by the promoters of schistosome genes, including cathepsin L (125). Recently, Brindley and coworkers have described somatic transgenesis of $S$. mansoni facilitated by either the piggyBac transposon (126) or the murine leukemia retrovirus (127), including the first demonstration of integration of reporter genes into the chromosomes of a parasitic worm.

Proteomics, glycomics, and metabolomics. Proteomic analyses in parasitic helminths are not as advanced as genomic or transcriptomic studies, but these aspects, along with glycomics and metabolomics, have now begun to be addressed. For example, proteomic analysis of the tegument of adults, the excreted and secreted products of adults, and the egg shells of S. japonicum and S. mansoni have yielded several thousand proteins, many of which have been putatively identified and many of which are much more closely related to the orthologous host proteins than to orthologs of invertebrates $(102,128)$. Proteomic investigations have also revealed differences between the secretome of Trichinella spiralis and that of Trichinella pseudospiralis (129) as well as the plasticity of the proteome of gutdwelling nematodes in response to immunological pressure (130). Glycomics will provide information about the glycan structures of helminths that are known to mediate and modulate host immunological responses to these parasites $(131,132)$. Moreover, techniques from developmental biology and other disciplines, such as whole mount in situ hybridization (WISH) and gene microarrays, have now been established as crucial tools for investigating the function of helminth genes and proteins (133-135).

\section{Global control of helminthiases and clinical research imperatives}

Beginning with the widespread use of the drug diethylcarbamazine for the treatment of LF in China during the 1970s, the mass treatment of human populations with anthelminthic drugs, known as mass drug administration (MDA), has been a major approach to controlling human helminthiases in developing countries (13). During the late 1980s, the first public-private partnership was formed to provide MDA of ivermectin to African populations at risk for onchocerciasis (13), and since then the mainstay of global worm control has been MDA of anthelminthic drugs through the activities of public-private partnerships, using either drugs donated by multinational corporations or low-cost generic drugs (Table 2). An important stimulus for these partnerships has been a series of resolutions adopted at the World Health Assembly - the major decision-making body for the WHO, which meets annually and is attended by delegates from each member nation - calling for MDA to control or eliminate one or more helminth infections of global importance. Since 2006, several partnerships committed to MDA have formed an alliance, which is known as the Global Network for Neglected Tropical Diseases and aims to increase efficiencies and produce economies of scale by delivering a package of drugs that simultaneously targets the six most common human helminthiases (ascariasis, trichuriasis, hookworm, schistosomiasis, LF, and onchocerciasis) as well as trachoma (13). Because of its low cost and cost savings, integrated helminth control has become highly attractive to both global policy makers and donors.

In resource-poor settings with a substantial burden of helminthiases, it has become a common practice during MDA to treat individuals regardless of whether or not they are currently infected with the disease-causing parasitic worm. However, based on a mixed legacy of relying on drugs to control or eliminate a widespread infectious disease, such as malaria, there are concerns that emerging drug resistance or other factors could derail global efforts to implement MDA, integrated or otherwise (13). These concerns, together with a need to better understand a number of fundamental clinical and epidemiological aspects of human helminth infections as well as their interactions with geographically overlapping coinfections (e.g., malaria and HIV/AIDS) have created an urgency for stepped up clinical research activities as they relate to largescale helminth control. Such activities have focused around three major areas: first, a reexamination of the health impact of human helminthic infections, with particular interest in the effects of mono- and polyparasitism on childhood growth and development as well as their effects on pregnancy and birth outcomes (63, 136-140); second, large-scale monitoring and evaluation of MDA and integrated control, along with operational research with goals to improve the access of populations to anthelminthic drugs and to monitor for possible drug resistance (141-145); and third, the development of new tools to control helminth infections, that is, drugs, diagnostics, and vaccines $(19,146)$.

For purposes of MDA and integrated control efforts, current synthesis of the available study data has culminated in a recently 


\section{Table 2}

Major global helminthic disease control initiatives

\begin{tabular}{|c|c|c|c|}
\hline Disease(s) & Public-private partnership & Major drug or control tool & $\begin{array}{l}\text { Target date control } \\
\text { by WHA resolution }\end{array}$ \\
\hline LF & Global Alliance to Eliminate LF (GAELF) & $\begin{array}{l}\text { Diethylcarbamazine and albendazole; } \\
\text { ivermectin and albendazole }\end{array}$ & 2020 \\
\hline Onchocerciasis & $\begin{array}{l}\text { an Programme for Onchocerciasis Control (APOC); } \\
\text { erciasis Elimination Program of the Americas (OEPA) }\end{array}$ & Ivermectin & 2010 \\
\hline Schistosomiasis & $\begin{array}{l}\text { Schistosomiasis Control Initiative (SCI); } \\
\text { Partnership for Parasite Control (PPC) }\end{array}$ & Praziquantel & 2010 \\
\hline $\begin{array}{l}\text { Soil-transmitted } \\
\text { helminth infections }\end{array}$ & $\begin{array}{l}\text { Partnership for Parasite Control (PPC); } \\
\text { Schistosomiasis Control Initiative (SCI); } \\
\text { Human Hookworm Vaccine Initiative (HHVI) }\end{array}$ & Albendazole or mebendazole & 2010 \\
\hline $\begin{array}{l}\text { Ascariasis, trichuriasis, } \\
\text { hookworm, schistosomiasis, LF, } \\
\text { onchocerciasis, and trachoma }\end{array}$ & $\begin{array}{c}\text { Global Network for Neglected Tropical } \\
\text { Diseases (GNNTDC) }\end{array}$ & $\begin{array}{l}\text { mectin or diethylcarbamazine; praziquantel; } \\
\text { albendazole or mebendazole; } \\
\text { and azithromycin }\end{array}$ & 2015 \\
\hline
\end{tabular}

issued set of WHO guidelines (147). Parallel studies are examining the added benefits of micronutrient supplementation, particularly in combating the anemias associated with hookworm and schistosomiasis $(148,149)$. Other studies are specifically examining the impact of infection among women who are pregnant and women of child-bearing age, risk groups who are often excluded from MDA because of fears of fetal toxicity $(136,150,151)$, or they are examining the early developmental effects of helminthic infections among preschool children (152). Despite the evident success of MDA, limitations have been observed in the effectiveness of treatment in some field settings, and concern has been raised about the potential for emergence of resistance to the mainstay drugs of MDA programs, including the benzimidazole anthelminthic, used predominantly to combat soil-transmitted helminth infections, and ivermectin, which is used to combat filarial infections $(146,153-158)$. However, with the exceptions of $\beta$-tubulin as a biomarker to determine nematode resistance to the benzimidazoles mebendazole and albendazole (159) and possibly ABC transporters and $\beta$-tubulin as biomarkers for determining resistance to ivermectin and other macrocyclic lactones (160), we lack robust biomarkers for detecting resistance to most anthelminthic drugs.

Despite the remarkable successes of MDA programs, disease elimination is often not obtained in highly endemic areas, and research on new drugs (and means for their successful implementation) is clearly needed to maintain and expand effective control. In the coming decade, the mining of newly completed helminth genomes should help to facilitate the discovery of new anthelminthic drugs. In the meantime, recent animal and clinical studies have documented the efficacy of artemether compounds for treating the early phases of schistosome infection (161) and a new broad-spectrum agent known as tribendimidine for treating soil-transmitted helminth infections (162). New classes of cysteine protease inhibitors are being developed as anthelminthic and antiprotozoal drugs (163), and other studies have indicated the efficacy of triclabendazole for infection with the difficult to treat liver fluke Fasciola hepatica $(164,165)$. Human and animal studies also suggest that tribendimidine will prove effective against food-borne flukes (166) and the drug moxidectin might become an alternative to ivermectin for the treatment of filarial infections (167). Finally, based on the observation that the filarial parasites
Wuchereria bancrofti and Onchocerca volvulus harbor bacterial endosymbionts of the genus Wolbachia and depend on these endosymbionts for normal metabolic and reproductive activities, there is excitement about the development and use of antibiotics to target adult filarial worms (168). This is of particular importance because to date there is no effective drug that targets the adult stage of these parasitic worms.

In parallel with drug discovery efforts are two anthelminthic vaccine development efforts, each conducted by a dedicated product-development partnership. For hookworm, the high rate of drug failure for single-dose mebendazole as well as rapid reinfection and the possible emergence of drug-resistant parasites have stimulated efforts by the Human Hookworm Vaccine Initiative to develop a bivalent vaccine comprised of recombinant hookworm vaccine antigens that target the infective larval stages and adult blood-feeding stages $(146,169,170)$. Currently, one larval antigen is being evaluated in a phase 1 clinical trial in a region of Brazil that is endemic for hookworm and a second adult hookworm antigen is about to enter into clinical trials (170). Related antigens are also being used to develop recombinant onchocerciasis vaccines (171). In addition, the Institut Pasteur is conducting clinical trials in sub-Saharan Africa using a recombinant vaccine (encoding a glutathione $S$-transferase) to protect against infection with S. haematobium (172). A surface protein encoding a novel tetraspanin is also awaiting early development as a vaccine to protect against infection with S. mansoni (173). Ultimately, it is anticipated that anthelminthic vaccines are likely to be used alongside drugs in an integrated program linking vaccination with chemotherapy $(146,174)$.

\section{Concluding comments}

The recent developments in molecular and medical helminthology could relatively soon be translated into a new generation of anthelminthic therapeutics. Based on advances in schistosome genomics, a family of promising schistosome vaccine antigens has already been identified (173) and ultimately could be formulated into a schistosomiasis vaccine. Such a vaccine could be used together with a hookworm vaccine. At the same time, the recently revealed importance of Wolbachia endosymbionts is helping to launch new drug discovery efforts for the treatment of LF and onchocerciasis 
as well as treatment programs based on either existing or new antibiotics (168). The recent completion of the filarial genome might soon yield additional drug targets (105). Revelations within the last five years about the impact of helminthiases on the transmission of the pathogens that cause malaria and HIV/AIDS and on the progression of these diseases (12), suggest that deworming and other large-scale anthelminthic measures might promote important "back-door" approaches for controlling them $(12,13)$.

At the same time, our enthusiasm for seeing helminthological science translate into new interventions needs to be tempered because of some sobering elements. First, despite these new advances, overall, we are still at a relatively nascent stage of helminth translational research and development. The development of in vitro methodologies and high throughput technologies for studying helminths has not, for the most part, kept up with the generation of parasitic helminth genomic and other bioinformatic databases. Until there is greater investment of scientific horsepower and funds into fundamental helminthology research, we are still a way off using the reverse genomic and vaccinology approaches that have benefited modern bacteriology. Second, because worm infections occur almost exclusively among the poorest people of the world, there are no market incentives for mining bioinformatic databases for anthelminthic drug and vaccine discovery. Therefore, unless there is financial innovation for the poor or a paradigm shift by the multinationals in orphan drug development for developing countries, we must either rely on the existing veterinary pipeline of new anthelminthics feeding into the human pipeline or we need to expand the role of nonprofit product-development partnerships $(4,13,146)$. Critical to the success of new anthelminthic interventions is likely be North-South partnerships with so-called "innovative developing countries," i.e., economically disadvantaged nations endemic for neglected tropical diseases such as Brazil, China, and India, which have nonetheless achieved a high level of biotechnological sophistication (175). Third, until vaccines or new drugs are developed for human helminthiases, we must rely on a tiny pharmacopeia of existing drugs. Currently, integrated control of the six most common human helminthiases, which affect approximately one billion people in developing countries, depends on the availability and effectiveness of just four drugs - albendazole, mebendazole, praziquantel, and ivermectin. Adequate resources need to be set aside for the development of genetic resistance markers and for careful monitoring and evaluation of large scale programs if we are to preserve these agents as public health control tools. Finally, in the absence adequate of new support for helminthology training, we risk losing a generation of whole-organism scientists who have the skills to study both the parasites and the diseases they cause. Because of the enormous benefits in human capital that would result from investing in the study of fundamental and translational helminthology and because access to anthelminthic interventions has now been recognized as a fundamental human right (176), we believe that now, more than ever, the scientific and global public health communities should address the neglected status of the helminthiases and prioritize their study.

\section{Acknowledgments}

The authors acknowledge with gratitude the editorial assistance of Sophia Raff and Julie Ost. The authors are supported by grants from the Sabin Vaccine Institute and the Bill and Melinda Gates Foundation (P.J. Hotez and J.M. Bethony), Geneva Global (P.J. Hotez), and National Institute of Allergy and Infectious Diseases, NIH (P.J. Hotez, P.J. Brindley, C.H. King, E.J. Pearce, and J.M. Bethony).

Address correspondence to: Peter J. Hotez, Department of Microbiology, Immunology, and Tropical Medicine, George Washington University School of Medicine and Health Sciences, 2300 I Street NW, Washington, DC 20037, USA. Phone: (202) 994-3532; Fax: (202) 994-2913; E-mail: PHotez@gwu.edu.
1. Faust, E.C., Russell, P.F., and Jung, R.C. 1970. Craig and Faust's clinical parasitology. 8th edition. Philadelphia, Lea \& Febiger. p. 251.

2. Cox, F.E.G. 2002. History of human parasitology. Clin. Microbiol. Rev. 15:595-612.

3. Hotez, P.J., Ottesen, E., Fenwick, A., and Molyneux, D. 2006. The neglected tropical diseases: the ancient afflictions of stigma and poverty and the prospects for their control and elimination. $A d v$. Exp. Med. Biol. 582:23-33.

4. Hotez, P.J. 2008. Forgotten people and forgotten diseases, the neglected tropical diseases and their impact on global health and development. ASM Press. In press.

5. Hotez, P.J. 2002. China's hookworms [abstract]. China O. 172:1029-1041.

6. Farley, J. 1991. Bilharzia, a history of imperial tropical medicine. Cambridge University Press. Cambridge, United Kingdom. 359 pp.

7. Kernan, F.A.J. 1959 April. The blood fluke that saved Formosa. Harper's Magazine. 45-47.

8. Stoll, N.R. 1999. This wormy world. J. Parasitol. 85:392-396.

9. de Silva, N.R., et al. 2003. Soil-transmitted helminth infections: updating the global picture. Trends Parasitol. 19:547-551.

10. Steinmann, P., Keiser, J., Bos, R., Tanner, M., and Utzinger, J. 2006. Schistosomiasis and water resources development: systematic review, metaanalysis, and estimates of people at risk. Lancet Infect. Dis. 6:411-425.

11. Ottesen, E.A. 2006. Lymphatic filariasis: treatment, control and elimination. Adv. Parasitol. 61:395-441.
12. Hotez, P.J., et al. 2006. Incorporating a rapidimpact package for neglected tropical diseases with programs for HIV/AIDS, tuberculosis, and malaria. PLoS Med. 3:e102.

13. Hotez, P.J., et al. 2007. Control of neglected tropical diseases. N. Engl. J. Med. 357:1018-1027.

14. Crompton, D.W., and Nesheim, M.C. 2002. Nutritional impact of intestinal helminthiasis during the human life cycle. Ann. Rev. Nutr. 22:35-59.

15. Miguel, E.A., and Kremer, M. 2003. Worms: identifying impacts on education and health in the presence of treatment externalities. Econometrica. 72:159-217.

16. Bleakley, H. 2007. Disease and development: evidence from hookworm eradication in the American South. Q. J. Econ. 122:73-112.

17. Christian, P., Khatry, S.K., and West, J.P. 2004. Antenatal anthelminthic treatment, Birth weight, and infant survival in rural Nepal. Lancet. 364:981-983.

18. Perera, M., Whitehead, M., Molyneux, D., Weerasooriya, M., and Gunatilleke, G. 2007. Neglected patients in neglected disease? Qualitative study of lymphatic filariasis. PLoS Negl. Trop. Dis. 1:e128.

19. Hotez, P.J., and Ferris, M.T. 2006. The antipoverty vaccines. Vaccine. 24:5787-5799.

20. Druilhe, P., Tall, A., and Sokhna, C. 2005. Worms can worsen malaria: towards a new means to roll back malaria? Trends Parasitol. 21:359-362.

21. Brooker, S., et al. 2006. The co-distribution of Plasmodium falciparum and hookworm among African schoolchildren. Malaria J. 5:99.

22. Brooker, S., et al. 2008. Epidemiology of Plasmodium-helminth co-infection in Africa: population at risk, potential impact on anemia, and prospects for combining control. Am. J. Trop. Med. Hyg. In press.

23. Borkow, G., and Bentwich, Z. 2006. HIV and helminth co-infection. Parasite Immunol. 28:605-612.

24. Secor, W.E. 2006. Interactions between schistosomiasis and infection with HIV-1. Parasite Immunol. 28:597-603.

25. Kjetland, E.F., et al. 2006. Association between genital schistosomiasis and HIV in rural Zimbabwean women. AIDS. 20:593-600.

26. Gallagher, M., et al. 2005. The effects of maternal helminth and malaria infections on mother-tochild HIV transmission. AIDS. 19:1849-1855.

27. Chirac, P., and Torreele, E. 2006. Global framework on essential health R\&D. Lancet. 367:1560-1561.

28. Fletcher, R.H., and Fletcher, S.W. 2005. Clinical epidemiology: the essentials. 4th edition. Lippincott, Williams, \& Wilkins. Philadelphia, Pennsylvania, USA. 288 pp.

29. Anderson, R.M. 1982. Population dynamics of infectious diseases: theory and applications. Chapman and Hall. London, United Kingdom. 377 pp.

30. Montresor, A., Crompton, D.W.T., Bundy, D.A.P., Hall, A., and Savioli, L. 1998. Guidelines for the evaluation of soil-transmitted helminthiasis and schistosiasis at community level. http://whqlibdoc. who.int/hq/1998/WHO_CTD_SIP_98.1.pdf

31. Brooker, S. 2007. Spatial epidemiology of human schistosomiasis in Africa: risk models, transmission dynamics and control. Trans. R. Soc. Trop. Med. Hyg. 101:1-8.

32. Sturrock, R.F. 1993. The intermediate hosts and host-parasite relationships. In Human Schistosomia- 
sis. P. Jordan, G. Webbe, and R.F. Sturrock, editors. $\mathrm{CAB}$ International. Wallingford, United Kingdom. 33-85.

33. Bockarie, M.J., and Davies, J.B. 1990. The transmission of onchocerciasis at a forest village in Sierra Leone. II. Man-fly contact, human activity and exposure to transmission. Ann. Trop. Med. Parasitol. 84:599-605

34. Bockarie, M.J., Davies, J.B., Thomson, M.C., and Morgan, H.G. 1990. The transmission of onchocerciasis at a forest village in Sierra Leone. I. Simulium damnosum s.l. biting densities and infection with Onchocerca volvulus at five representative sites. Ann. Trop. Med. Parasitol. 84:587-597.

35. Brooker, S., et al. 2003. Mapping soil-transmitted helminths in Southeast Asia and implications for parasite control. Southeast Asian J. Trop. Med. Public Health. 34:24-36.

36. Appleton, C.C., and Gouws, E. 1996. The distribution of common intestinal nematodes along an altitudinal transect in KwaZulu-Natal, South Africa. Ann. Trop. Med. Parasitol. 90:181-188.

37. Kariuki, H.C., et al. 2004. Distribution patterns and cercarial shedding of Bulinus nasutus and other snails in the Msambweni area, Coast Province, Kenya. Am. J. Trop. Med. Hyg. 70:449-456.

38. Woolhouse, M.E., et al. 1997. Heterogeneities in the transmission of infectious agents: implications for the design of control programs. Proc. Natl. Acad. Sci. U. S. A. 94:338-342.

39. Anderson, R.M., and May, R.M. 1991. Infectious diseases of humans: dynamics and control. Oxford Science Publications. Oxford, United Kingdom. 768 pp.

40. Albonico, M., Crompton, D.W., and Savioli, L. 1999. Control strategies for human intestinal nematode infections. Adv. Parasitol. 42:277-341.

41. Schad, G.A., and Anderson, R.M. 1985. Predisposition to hookworm infection in humans. Science. 228:1537-1540.

42. Chan, M.S., Guyatt, H.L., Bundy, D.A.P., and Medley, G.F. 1996. Dynamic models of schistosomiasis morbidity. Am. J. Trop. Med. Hyg. 55:52-62.

43. Gilles, H. 1996. Soil-transmitted helminths (geohelminths). In Manson's tropical diseases. G. Cook, editor. W.B. Saunders. London, United Kingdom. 1369-1412.

44. Woolhouse, M.E., Taylor, P., Matanhire, D., and Chandiwana, S.K. 1991. Acquired immunity and epidemiology of Schistosoma haematobium. Nature. 351:757-759.

45. Bethony, J., et al. 2002. Emerging patterns of hookworm infection: influence of aging on the intensity of Necator infection in Hainan Province, People's Republic of China. CID. 35:1366-1344.

46. Woolhouse, M.E. 1998. Patterns in parasite epidemiology: the peak shift. Parasitol. Today. 14:428-434.

47. Forrester, J.E., Scott, M.E., Bundy, D.A., and Golden, M.H. 1988. Clustering of Ascaris lumbricoides and Trichuris trichiura infections within households. Trans. R. Soc. Trop. Med. Hyg. 82:282-288.

48. Conway, D.J., Hall, A., Anwar, K.S., Rahman, M.L., and Bundy, D.A. 1995. Household aggregation of Strongyloides stercoralis infection in Bangladesh. Trans. R. Soc. Trop. Med. Hyg. 89:258-261.

49. Lindo, J.F., et al. 1995. Age-prevalence and household clustering of Strongyloides stercoralis infection in Jamaica. Parasitology. 110:97-102.

50. Forrester, J.E., Scott, M.E., Bundy, D.A.P., and Golden, M.H.N. 1990. Predisposition of individuals and families in Mexico to heavy infection with Ascaris lumbricoides and Trichuris trichiura. Trans. R. Soc. Trop. Med. Hyg. 84:272-276.

51. Walter, S.D. 1974. On the detection of household aggregation of disease. Biometrics. 30:525-538.

52. Ottesen, E.A., et al. 1981. Familial predisposition to filarial infection - not linked to HLA-A or-B locus specificities. Acta Trop. 38:205-216.

53. Bethony, J., et al. 2001. Exposure to Schistosoma mansoni infection in a rural area in Brazil. II: household risk factors. Trop. Med. Int. Health. 6:136-145.

54. Bethony, J., et al. 1999. Familial resemblance in humoral immune response to defined and crude Schistosoma mansoni antigens in an endemic area in Brazil. J. Infect. Dis. 180:1665-1673.

55. Quinnell, R.J. 2003. Genetics of susceptibility to human helminth infection. Int. J. Parasitol. 33:1219-1231.

56. Marquet, S., et al. 1996. Genetic localization of a locus controlling the intensity of infection by Schistosoma mansoni on chromosome 5q31-q33. Nat. Genet. 14:181-184.

57. Müller-Myhsok, B., et al. 1997. Further evidence suggesting the presence of a locus, on human chromosome 5q31-q33, influencing the intensity of infection with Schistosoma mansoni. Am. J. Hum. Genet. 61:452-454.

58. Booth, M., et al. 1998. Associations among multiple geohelminth species infections in schoolchildren from Pemba Island. Parasitology. 116:85-93.

59. Needham, C., et al. 1998. Epidemiology of soiltransmitted nematode infections in Ha Nam Province, Vietnam. Trop. Med. Int. Health. 3:904-912.

60. Brooker, S., et al. 2000. Epidemiology of single and multiple species of helminth infections among school children in Busia District, Kenya. East Afr. Med. J. 77:157-161.

61. Tchuem Tchuente, L.A., Behnke, J.M., Gilbert, F.S., Southgate, V.R., and Vercruysse, J. 2003. Polyparasitism with Schistosoma haematobium and soiltransmitted helminth infections among school children in Loum, Cameroon. Trop. Med. Int. Health. 8:975-986.

62. Raso, G., et al. 2004. Multiple parasite infections and their relationship to self-reported morbidity in a community of rural Cote d'Ivoire. Int. J. Epidemiol. 33:1092-1102

63. Fleming, F.M., et al. 2006. Synergistic associations between hookworm and other helminth species in a rural community in Brazil. Trop. Med. Int. Health. 11:56-64.

64. Keiser, J., et al. 2002. Polyparasitism with Schistosoma mansoni, geohelminths, and intestinal protozoa in rural Cote d'Ivoire. J. Parasitol. 88:461-466.

65. Faulkner, H., et al. 2005. Associations between filarial and gastrointestinal nematodes. Trans. $R$. Soc. Trop. Med. Hyg. 99:301-312.

66. Mwangi, T.W., Bethony, J.M., and Brooker, S. 2006 Malaria and helminth interactions in humans: an epidemiological viewpoint. Ann. Trop. Med. Parasitol. 100:551-570.

67. King, C.H. 2007. Lifting the burden of schistosomiasis - defining elements of infection-associated disease and the benefits of anti-parasite treatment. J. Infect. Dis. 196:653-655.

68. Bethony, J., et al. 2006. Soil-transmitted helminth infections: ascariasis, trichuriasis, and hookworm Lancet. 367:1521-1532.

69. Budke, C.M., Jiamin, Q., Qian, W., and Torgerson, P.R. 2005. Economic effects of echinococcosis in a disease-endemic region of the Tibetan Plateau. Am. J. Trop. Med. Hyg. 73:2-10.

70. King, C.H., and Dangerfield-Cha, M. 2008. The unacknowledged impact of chronic schistosomiasis. Chronic Illness. In press.

71. Murray, C.J.L., and Lopez, A.D., editors. 1996. The global burden of disease: a comprehensive assessment of mortality and disability from diseases, injuries, and risk factors in 1990 and projected to 2020. Harvard School of Public Health. Cambridge, Massachusetts, USA. 990 pp.

72. Anand, S., and Hanson, K. 1997. Disability-adjusted life years: a critical review. J. Health Econ. 16:685-702.

73. Mathers, C.D., Ezzati, M., and Lopez, A.D. 2007. Measuring the burden of neglected tropical diseases: the global burden of disease framework. PLoS Negl. Trop. Dis. 1:e114.
74. Jia, T.W., et al. 2007. Assessment of the age-specific disability weight of chronic schistosomiasis japonica. Bull. World Health Organ. 85:458-465.

75. Budke, C.M., Jiamin, Q., Zinsstag, J., Qian, W., and Torgerson, P.R. 2004. Use of disability adjusted life years in the estimation of the disease burden of echinococcosis for a high endemic region of the Tibetan plateau. Am. J. Trop. Med. Hyg. 71:56-64.

76. Gold, M.R., Siegel, J.E., Russell, L.B., and Weinstein, M.C. 1996. Cost effectiveness in health and medicine. Oxford University Press. New York, New York, USA. 425 pp.

77. Voehringer, D., Shinkai, K., and Locksley, R.M. 2004. Type 2 immunity reflects orchestrated recruitment of cells committed to IL-4 production. Immunity. 20:267-277.

78. Finkelman, F.D., et al. 2004. Interleukin-4- and interleukin-13-mediated host protection against intestinal nematode parasites. Immunol. Rev. 201:139-155.

79. Voehringer, D., Reese, T.A., Huang, X., Shinkai, K., and Locksley, R.M. 2006. Type 2 immunity is controlled by IL-4/IL-13 expression in hematopoietic non-eosinophil cells of the innate immune system. J. Exp. Med. 203:1435-1446.

80. Herbert, D.R., et al. 2004. Alternative macrophage activation is essential for survival during schistosomiasis and downmodulates $T$ helper 1 responses and immunopathology. Immunity. 20:623-635.

81. Wynn, T.A., Thompson, R.W., Cheever, A.W., and Mentink-Kane, M.M. 2004. Immunopathogenesis of schistosomiasis. Immunol. Rev. 201:156-167.

82. Loke, P., et al. 2007. Alternative activation is an innate response to injury that requires CD4+ T cells to be sustained during chronic infection. J. Immunol. 179:3926-3936.

83. Reinhardt, R.L., Kang, S.J., Liang, H.E., and Locksley, R.M. 2006. T helper cell effector fates - who, how and where? Curr. Opin. Immunol. 18:271-277.

84. Stockinger, B., and Veldhoen, M. 2007. Differentiation and function of Th17 T cells. Curr. Opin. Immunol. 19:281-286.

85. Fort, M.M., et al. 2001. IL-25 induces IL-4, IL-5, and IL-13 and Th2-associated pathologies in vivo. Immunity. 15:985-995.

86. Dillon, S.R., et al. 2004. Interleukin 31, a cytokine produced by activated $\mathrm{T}$ cells, induces dermatitis in mice. Nat. Immunol. 5:752-760.

87. Liu, Y.J., et al. 2007. TSLP: an epithelial cell cytokine that regulates $\mathrm{T}$ cell differentiation by conditioning dendritic cell maturation. Annu. Rev. Immunol. 25:193-219.

88. Nair, M.G., Guild, K.J., and Artis, D. 2006. Novel effector molecules in type 2 inflammation: lessons drawn from helminth infection and allergy. J. Immunol. 177:1393-1399.

89. Kreider, T., Anthony, R.M., Urban, J.F., and Gause, W.C. 2007. Alternatively activated macrophages in helminth infections. Curr. Opin. Immunol. 19:448-453.

90. Anthony, R.M., et al. 2006. Memory T(H)2 cells induce alternatively activated macrophages to mediate protection against nematode parasites. Nat. Med. 12:955-960.

91. Fallon, P.G., et al. 2006. Identification of an interleukin (IL)-25-dependent cell population that provides IL-4, IL-5, and IL-13 at the onset of helminth expulsion. J. Exp. Med. 203:1105-1116.

92. Owyang, A.M., et al. 2006. Interleukin 25 regulates type 2 cytokine-dependent immunity and limits chronic inflammation in the gastrointestinal tract. J. Exp. Med. 203:843-849.

93. Perrigoue, J.G., et al. 2007. IL-31-IL-31R interactions negatively regulate type 2 inflammation in the lung. J. Exp. Med. 204:481-487.

94. Zaph, C., et al. 2007. Epithelial-cell-intrinsic IKKbeta expression regulates intestinal immune homeostasis. Nature. 446:552-556. 
95. Maizels, R.M., et al. 2004. Helminth parasites masters of regulation. Immunol. Rev 201:89-116.

96. Harnett, W., and Harnett, M.M. 2006. Filarial nematode secreted product ES-62 is an anti-inflammatory agent: therapeutic potential of small molecule derivatives and ES-62 peptide mimetics. Clin. Exp. Pharmacol. Physiol. 33:511-518.

97. Wilson, M.S., and Maizels, R.M. 2006. Regulatory $T$ cells induced by parasites and the modulation of allergic responses. Chem. Immunol. Allergy. 90:176-195.

98. Smits, H.H., and Yazdanbakhsh, M. 2007. Chronic helminth infections modulate allergen-specific immune responses: protection against development of allergic disorders? Ann. Med. 39:428-439.

99. Haas, B.J., et al. 2007. Schistosoma mansoni genome: closing in on a final gene set. Exp. Parasitol. 117:225-228.

100.Hu, W., et al. 2003. Evolutionary and biomedical implications of a Schistosoma japonicum complementary DNA resource. Nat. Genet. 35:139-147.

101.Verjovski-Almeida, S., et al. 2003. Transcriptome analysis of the acoelomate human parasite Schistosoma mansoni. Nat. Genet. 35:148-157.

102.Liu, F., et al. 2006. Comparative transcriptomic and proteomic analyses of the human blood fluke, Schistosoma japonicum. PLoS Pathog. 2:e29.

103. Hirai, H., et al. 2000. Chromosomal differentiation of the Schistosoma japonicum complex. Int. J. Parasitol. 30:441-452.

104. Brindley, P.J. 2005. The molecular biology of schistosomes. Trend Parasitol. 21:533-536.

105. Ghedin, E., et al. 2007. The draft genome of the filarial nematode Brugia malayi. Science. 317:1756-1760.

106. Parkinson, J., et al. 2004. A transcriptomic analysis of the phylum Nematoda. Nat. Genet. 36:1259-1267.

107. Mitreva, M., et al. 2006. Codon usage patterns in Nematoda: analysis based on over 25 million codons in thirty-two species. Genome Biol. 7:R75.

108.Brooker, S., Bethony, J., and Hotez, P.J. 2004. Human hookworm infection in the 21 st century. Adv. Parasitol. 58:197-288.

109.Giugliano, R.P., et al. 2007. Recombinant nematode anticoagulant protein $\mathrm{c} 2$ in patients with nonST-segment elevation acute coronary syndrome: the ANTHEM-TIMI-32 trial. J. Am. Coll. Cardiol. 49:2398-2407.

110. Mahajan, A.L., et al. 2006. Progressive tissue injury in burns is reduced by rNAPc2. Burns. 32:957-963.

111. Geisbert, T.W., et al. 2003. Treatment of Ebola virus infection with a recombinant inhibitor of factor $\mathrm{VII} /$ /tissue factor: a study in rhesus monkeys. Lancet. 362:1953-1958.

112. Krams, M., et al. 2003. Acute stroke therapy by inhibition of neutrophils (ASTIN): an adaptive doseresponse study of UK-279,276 in acute ischemic stroke. Stroke. 34:2543-2548.

113.Skelly, P.J., Da'dara, A., and Harn, D.A. 2003. Suppression of cathepsin B expression in Schistosoma mansoni by RNA interference. Int. J. Parasitol. 33:363-369.

114.Krautz-Peterson, G., Radwanska, M., Ndegwa, D., Shoemaker, C.B., and Skelly, P.J. 2007. Optimizing gene suppression in schistosomes using RNA interference. Mol. Biochem. Parasitol. 153:194-202.

115.Correnti, J.M., Brindley, P.J., and Pearce, E.J. 2005. Long-term suppression of cathepsin B levels by RNA interference retards schistosome growth. Mol. Biochem. Parasitol. 143:209-215.

116. Dinguirard, N., and Yoshino, T.P. 2006. Potential role of a CD36-like class B scavenger receptor in the binding of modified low-density lipoprotein (acLDL) to the tegumental surface of Schistosoma mansoni sporocysts. Mol. Biochem. Parasitol. 146:219-230.

117. Delcroix, M., et al. 2006. A multienzyme network functions in intestinal protein digestion by a platyhelminth parasite. J. Biol. Chem. 281:39316-39329.

118. Freitas, T.C., Jung, E., and Pearce, E.J. 2007. TGF- beta signaling controls embryo development in the parasitic flatworm Schistosoma mansoni. PLoS Pathogens. 3:e52

119.Lustigman, S., Zhang, J., Liu, J., Oksov, Y., and Hashmi, S. 2004. RNA interference targeting cathepsin L and Z-like cysteine proteases of Onchocerca volvulus confirmed their essential function during L3 molting. Mol. Biochem. Parasitol. 138:165-170.

120. Knox, D.P., Geldhof, P., Visser, A., and Britton, C. 2007. RNA interference in parasitic nematodes of animals: a reality check? Trends Parasitol. 23:105-107.

121.Viney, M.E., and Thompson, F.J. 2008. Two hypotheses to explain why RNA interference does not work in animal parasitic nematodes. Int. J. Parasitol. 38:43-47.

122.Higasi, T.B., Shu, L., and Unnasch, T.R. 2004. Development and transfection of short-term primary cell cultures from Brugia malayi. Mol. Biochem. Parasitol. 137:345-348.

123.Li, X., et al. 2006. Successful transgenesis of the parasitic nematode Strongyloides stercoralis requires endogenous non-coding control elements. Int. J. Parasitol. 36:671-679.

124.Grant, W.N., et al. 2006. Heritable transgenesis of Parastrongyloides trichosuri: a nematode parasite of mammals. Int. J. Parasitol. 36:475-483.

125. Wippersteg, V., et al. 2005. Biolistic transformation of Schistosoma mansoni with 5 ' flanking regions of two peptidase genes promotes tissue-specific expression. Int. J. Parasitol. 35:583-589.

126. Morales, M.E., et al. 2007. PiggyBac transposon mediated transgenesis of the human blood fluke, Schistosoma mansoni. FASEB J. 21:3479-3489.

127. Kines, K.J., et al. 2006. Transduction of Schistosoma mansoni by vesicular stomatitis virus glycoproteinpseudotyped Moloney murine leukemia retrovirus. Exp. Parasitol. 112:209-220.

128. Braschi, S., Curwen, R.S., Ashton, P.D., VerjovskiAlmeida, S., and Wilson, R.A. 2006. The tegument surface membranes of the human blood parasite Schistosoma mansoni: a proteomic analysis after differential extraction. Proteomics. 6:1471-1482.

129. Robinson, M.W., Grieg, R., Beattie, K.A., Lamont, D.J., and Connolly, B. 2005. Comparative analysis of the excretory-secretory proteome of the muscle larva of Trichinella pseudospiralis and Trichinella spiralis. Int. J. Parasitol. 37:139-148.

130.Morgan, C., et al. 2006. Plasticity demonstrated in the proteome of a parasitic nematode within the intestine of different host strains. Proteomics. 6:4633-4645

131.Hokke, C.H., Deelder, A.M., Hoffmann, K.F., and Wuhrer, M. 2007. Glycomics-driven discoveries in schistosome research. Exp. Parasitol. 117:275-283.

132.Jang-Lee, J., et al. 2007. Glycomics analysis of Schistosoma mansoni egg and cercarial secretions. Mol. Cell. Proteomics. 6:1485-1499.

133.Moser, J.M., Freitas, T., Arasu, P., and Gibson, G. 2005. Gene expression profiles associated with the transition to parasitism in Ancylostoma caninum larvae. Mol. Biochem. Parasitol. 143:39-48.

134.Gobert, G.N., et al. 2006. Transcriptomics tool for the human Schistosoma blood flukes using microarray gene expression profiling. Exp. Parasitol. 114:160-172.

135.Dillon, G.P., Illes, J.C., Isaacs, H.V., and Wilson, R.A. 2007. Patterns of gene expression in schistosomes: localization by whole mount in situ hybridization. Parasitology. 134:1589-1597.

136. Friedman, J.F., Mital, P., Kanzaria, H.K., Olds, G.R., and Kurtis, J.D. 2007. Schistosomiasis and pregnancy. Trends Parasitol. 23:159-164.

137. Assis, A.M., et al. 2004. Childhood stunting in Northeast Brazil: the role of Schistosoma manson infection and inadequate dietary intake. Eur. J. Clin. Nutr. 58:1022-1029.

138.Casapia, M., Joseph, S.A., Nunez, C., Rahme, E., and Gyorkos, T.W. 2006. Parasite risk factors for stunt- ing in grade 5 students in a community of extreme poverty in Peru. Int. J. Parasitol. 36:741-747.

139. Larocque, R., et al. 2006. A double-blind randomized controlled trial of antenatal mebendazole to reduce low birth weight in a hookworm-endemic area of Peru. Trop. Med. Int. Health. 11:1485-1495.

140.Ajanga, A., et al. 2006. Schistosoma mansoni in pregnancy and associations with anaemia in northwest Tanzania. Trans. R. Soc. Trop. Med. Hyg. 100:59-63.

141.Molyneux, D.H., Hotez, P.J., and Fenwick, A. 2005. "Rapid-impact interventions": how a policy of integrated control for Africa's neglected tropical diseases could benefit the poor. PLoS Med. 2:e336.

142.Fenwick, A. 2006. New initiatives against Africa's worms. Trans. R. Soc. Trop. Med. Hyg. 100:200-207.

143.Richards, F.O., Jr., Eigege, A., Miri, E.S., Jinadu, M.Y., and Hopkins, D.R. 2006. Integration of mass drug administration programmes in Nigeria: the challenge of schistosomiasis. Bull. World Health Organ. 84:673-676.

144.Boia, M.N., et al. 2006. Mass treatment for intestinal helminthiasis control in an Amazonian endemic area in Brazil. Rev. Inst. Med. Trop. Sao Paulo. 48:189-195.

145. King, C.H. 2006. Long-term outcomes of schoolbased treatment for control of urinary schistosomiasis: a review of experience in Coast Province, Kenya. Mem. Inst. Oswaldo Cruz. 101:299-306.

146. Hotez, P.J., et al. 2006. New technologies for the control of human hookworm infection. Trends Parasitol. 22:327-331.

147. WHO. 2006. Preventive chemotherapy in human helminthiasis: coordinated use of anthelminthic drugs in control interventions: a manual for health professionals and programme managers. http://whqlibdoc. who.int/publications/2006/9241547103_eng.pdf.

148.Hall, A. 2007. Micronutrient supplements for children after deworming. Lancet Infect. Dis. 7:297-302.

149. de Silva, N.R. 2003. Impact of mass chemotherapy on the morbidity due to soil-transmitted nematodes. Acta Trop. 86:197-214.

150.Gyorkos, T.W., Larocque, R., Casapia, M., and Gotuzzo, E. 2006. Lack of risk of adverse birth outcomes after deworming in pregnant women. Pediatr. Infect. Dis. J. 25:791-794.

151.Allen, H.E., Crompton, D.W., de Silva, N., LoVerde, P.T., and Olds, G.R. 2002. New policies for using anthelmintics in high risk groups. Trends Parasitol. 18:381-382.

152.Stothard, J.R., and Gabrielli, A.F. 2007. Schistosomiasis in African infants and preschool children: to treat or not to treat? Trends Parasitol. 23:83-86.

153. Flohr, C., et al. 2007. Low efficacy of mebendazole against hookworm in Vietnam: two randomized controlled trials. Am. J. Trop. Med. Hyg. 76:732-736.

154.Basanez, M.G., et al. 2006. River blindness: a success story under threat? PLoS Med. 3:e371.

155.Geerts, S., and Gryseels, B. 2000. Drug resistance in human helminths: current situation and lessons from livestock. Clin. Microbiol. Rev. 13:207-222.

156.Albonico, M., Engels, D., and Savioli, L. 2004. Monitoring drug efficacy and early detection of drug resistance in human soil-transmitted nematodes: a pressing public health agenda for helminth control. Int. J. Parasitol. 34:1205-1210.

157. Osei-Atweneboana, M.Y., Eng, J.K.L., Boakye, D.A., Gyapong, J.O., and Prichard, R.K. 2007. Prevalence and intensity of Onchocerca volvulus infection and efficacy of ivermectine after 19 years of treatment in endemic communities in Ghana. Lancet. 369:2021-2029.

158. Bourguinat, C., et al. 2007. Genetic selection of low fertile Onchocerca volvulus by ivermectin treatment. PLoS Negl. Trop. Dis. 30:e72.

159.Prichard, R.K. 2007. Markers for benzimidazole resistance in human parasitic nematodes? Parasitology. 134:1087-1092.

160.Prichard, R.K., and Roulet, A. 2007. ABC transport- 
ers and beta-tubulin in macrocyclic lactone resistance: prospects for marker development. Parasitology. 134:1123-1132.

161.Utzinger, J., Xiao, S.H., Tanner, M., and Keiser, J. 2007. Artemisinins for schistosomiasis and beyond. Curr. Opin. Investig. Drugs. 8:105-116.

162.Xiao, S.H., Hui-Ming, W., Tanner, M., Utzinger, J. and Chong, W. 2005. Tribendimidine: a promising, safe and broad-spectrum anthelmintic agent from China. Acta Trop. 94:1-14.

163.Abdulla, M.H., Lim, K.C., Sajid, M., McKerrow, J.H., and Caffrey, C.R. 2007. Schistosomiasis mansoni: novel chemotherapy using a cysteine protease inhibitor. PLoS Med. 4:e14.

164.Richter, J., Knipper, M., Gobels, K., and Haussinger, D. 2002. Fascioliasis. Current Treatment Options in Infectious Diseases. 4:313-317.

165. Keiser, J., Engels, D., Buscher, G., and Utzinger, J. 2005. Triclabendazole for the treatment of fascioliasis and paragonimiasis. Expert Opin. Investig. Drugs.
14:1513-1526.

166.Keiser, J., Shu-Hua, X., Chollet, J., Tanner, M., and Utzinger, J. 2007. Evaluation of the in vivo activity of tribendimidine against Schistosoma mansoni, Fasciola hepatica, Clonorchis sinensis, and Opisthorchis viverrini. Antimicrob. Agents Chemother. 51:1096-1098.

167. Cotreau, M.M., et al. 2003. The antiparasitic moxidectin: safety, tolerability, and pharmacokinetics in humans. J. Clin. Pharmacol. 43:1108-1115.

168.Taylor, M.J., et al. 2005. Macrofilaricidal activity after doxycycline treatment of Wuchereria bancrofti: a double-blind, randomised placebo-controlled trial. Lancet. 365:2116-2121.

169.Loukas, A., Bethony, J., Brooker, S., and Hotez, P. 2006. Hookworm vaccines: past, present, and future. Lancet Infect. Dis. 6:733-741.

170.Diemert, D., Bethony, J., and Hotez, P.J. 2008. Hookworm vaccines. Clin. Infect. Dis. 15:282-288.

171.Lustigman, S., James, E.R., Tawe, W., and Abraham, D. 2002. Towards a recombinant antigen vaccine against Onchocerca volvulus. Trends Parasitol. 18:135-141.

172.Capron, A., Riveau, G., Capron, M., and Trottein, F. 2005. Schistosomes: the road from host-parasite interactions to vaccines in clinical trials. Trends Parasitol. 21:143-149.

173. Tran, M.H., et al. 2006. Tetraspanins on the surface of Schistosoma mansoni are protective antigens against schistosomiasis. Nat. Med. 12:835-840.

174.Bergquist, N.R., Leonardo, L.R., and Mitchell, G.F. 2005. Vaccine-linked chemotherapy: can schistosomiasis control benefit from an integrated approach? Trends Parasitol. 21:112-117.

175.Morel, C.M., et al. 2005. Health innovation networks to help developing countries address neglected diseases. Science. 309:401-404.

176. Hunt, P. 2006. The human right to the highest attainable standard to health: new opportunities and challenges. Trans. R. Soc. Trop. Med. Hyg. 100:603-607. 